\title{
The maximum order of adjacency matrices of graphs with a given rank
}

\author{
W. H. Haemers • M. J. P. Peeters
}

Received: 28 October 2010 / Revised: 19 May 2011 / Accepted: 14 July 2011 /

Published online: 2 August 2011

(C) The Author(s) 2011. This article is published with open access at Springerlink.com

\begin{abstract}
We look for the maximum order $m(r)$ of the adjacency matrix $A$ of a graph $G$ with a fixed rank $r$, provided $A$ has no repeated rows or all-zero row. Akbari, Cameron and Khosrovshahi conjecture that $m(r)=2^{(r+2) / 2}-2$ if $r$ is even, and $m(r)=5 \cdot 2^{(r-3) / 2}-2$ if $r$ is odd. We prove the conjecture and characterize $G$ in the case that $G$ contains an induced subgraph $\frac{r}{2} K_{2}$ or $\frac{r-3}{2} K_{2}+K_{3}$.
\end{abstract}

Keywords Graph · Adjacency matrix

Mathematics Subject Classification (2000) 05B20 · 05C50

\section{Introduction}

In this paper we discus the problem of determining the maximum number of vertices of a graph in terms of the rank of its adjacency matrix. This problem is only properly defined if the matrix has no repeated rows. Given a graph we can duplicate a vertex arbitrarily often and add isolated vertices as many as we like without changing the rank. This motivates the following definition [2].

Definition 1 A graph is reduced if it has no isolated vertices and no two vertices have the same set of neighbors.

Although we find this definition more convenient, some authors [1,3] use 'reduced' purely for the condition that no two vertices have the same set of neighbors and allow the graph to have at most one isolated vertex. This explains why there is sometimes a difference of one in their results and ours.

This is one of several papers published together in Designs, Codes and Cryptography on the special topic: "Combinatorics - A Special Issue Dedicated to the 65th Birthday of Richard Wilson".

W. H. Haemers $(\varangle) \cdot$ M. J. P. Peeters

Department of Econometrics and Operations Research, Tilburg University, Tilburg, The Netherlands e-mail: haemers@uvt.nl 
Let $m(r)$ be the maximum number of vertices in a reduced graph with rank $r$ over $\mathbb{R}$. There are clearly no graphs of rank 1 , and the only reduced graphs of rank 2 and 3 are $K_{2}$ and $K_{3}$, respectively. The number $m(r)$ can be defined for any field and for all these cases there is an upper bound of $2^{r}-1$ (see Sect. 3). Godsil and Royle [2] prove that for the field $\mathbb{F}_{2}, m(r)$ is equal to this upper bound. In this paper we only consider $m(r)$ for the field of real numbers.

Kotlov and Lovász [3] improve the upper bound on $m(r)$ to $\mathcal{O}\left(2^{r / 2}\right)$. They also give a construction (Construction (a) in the next section) that transforms a reduced graph of rank $r$ on $n$ vertices into a reduced graph of rank $r+2$ on $2 n+2$ vertices.

Starting with $K_{2}$ (with $n=r=2$ ) or $K_{3}$ (with $n=r=3$ ) and recursively applying the construction leads to graphs of rank $r$ with $n(r)$ vertices where

$$
n(r)=\left\{\begin{array}{ll}
2^{(r+2) / 2}-2 & \text { if } r \text { is even, } \\
5 \cdot 2^{(r-3) / 2}-2 & \text { if } r \text { is odd, } r>1 .
\end{array} .\right.
$$

So we have $m(r) \geq n(r)$. Akbari et al. [1] give a second construction (Construction (b) in the next section) that transforms a reduced regular graph of rank $r$ on $n$ vertices with degree $\frac{n}{2}$ into a reduced regular graph of rank $r+2$ on $2 n+2$ vertices with degree $n+1$. For this construction one can start with $K_{2}$ or the complement of cube (which has $r=5$ ). In fact, at any step in this construction process, one can change to Construction (a). This leads to $\left\lfloor\frac{r}{2}\right\rfloor$ nonisomorphic graphs of rank $r$ with $n(r)$ vertices. The authors of [1] conjecture that these and only these graphs have the maximum number of vertices $m(r)$.

Conjecture If $r \geq 2$, then $m(r)=n(r)$, and the extremal graphs can be obtained from $K_{2}, K_{3}$ or the complement of the cube by Constructions $(a)$ and $(b)$.

The conjecture has been verified by computer up to $r=8$. In this paper we shall prove the conjecture for reduced graphs with rank $r$ that contain an induced subgraph $\frac{r}{2} K_{2}$ or $\frac{r-3}{2} K_{2}+K_{3}$. We also present two new constructions. These constructions, however, do not lead to a counter example for the conjecture.

\section{Constructions}

The lower bound $n(r)$ for $m(r)$ mentioned above, is based upon constructions that transform a reduced graph on $n$ vertices with rank $r$ into a reduced graph on $2 n+2$ vertices with rank $r+2$. Here we present four such constructions. The first construction is due to Kotlov and Lovász [3] and the second one to Akbari, Cameron and Khosrovshahi [1]. The other two seem to be new.

Construction (a) [1,3] Let $G$ be a reduced graph with $n$ vertices, adjacency matrix $A$ and rank $r$. Construct the graph $G^{a}$ with $2 n+2$ vertices and adjacency matrix:

$$
\left[\begin{array}{llll}
A & A & \mathbf{0} & \mathbf{0} \\
A & A & \mathbf{1} & \mathbf{0} \\
\mathbf{0} & \mathbf{1} & 0 & 1 \\
\mathbf{0} & \mathbf{0} & 1 & 0
\end{array}\right],
$$

where $\mathbf{0}$ and $\mathbf{1}$ are the all-zero and the all-one row or column vectors, respectively. It is straightforward to check that $G^{a}$ is reduced and has rank $r+2$.

Construction (b) [1] Let $G$ be a reduced regular graph with $n$ vertices with degree $\frac{n}{2}$, adjacency matrix $A$ and rank $r$. Construct the graph $G^{b}$ with $2 n+2$ vertices and adjacency matrix: 


$$
\left[\begin{array}{llll}
A & A & \mathbf{1} & \mathbf{0} \\
A & A & \mathbf{0} & \mathbf{1} \\
\mathbf{1} & \mathbf{0} & 0 & 1 \\
\mathbf{0} & \mathbf{1} & 1 & 0
\end{array}\right] .
$$

It is straightforward to check that $G^{b}$ is a reduced regular graph of rank $r+2$ with degree $n+1$.

Construction (c) Let $G$ be a reduced graph with $n$ vertices, adjacency matrix $A$ and rank $r$. Construct the graph $G^{c}$ with $2 n+2$ vertices and adjacency matrix:

$$
\left[\begin{array}{llll}
A & \bar{A} & \mathbf{1} & \mathbf{0} \\
\bar{A} & A & \mathbf{0} & \mathbf{1} \\
\mathbf{1} & \mathbf{0} & 0 & 1 \\
\mathbf{0} & \mathbf{1} & 1 & 0
\end{array}\right],
$$

where $\bar{A}=J-A$ for the all-one matrix $J$. It is straightforward to check that $G^{c}$ is a reduced regular graph of rank $r+2$ with degree $n+1$.

Construction (d) Let $G$ be a reduced regular graph with $n$ vertices with degree $\frac{n}{2}$, adjacency matrix $A$, and rank $r$. Construct the graph $G^{d}$ with $2 n+2$ vertices and adjacency matrix:

$$
\left[\begin{array}{llll}
A & \bar{A} & \mathbf{0} & \mathbf{0} \\
\bar{A} & A & \mathbf{1} & \mathbf{0} \\
\mathbf{0} & \mathbf{1} & 0 & 1 \\
\mathbf{0} & \mathbf{0} & 1 & 0
\end{array}\right] .
$$

It is straightforward to check that $G^{d}$ is a reduced graph with rank $r+2$.

Notice that Constructions (a) and (c) can be applied for any reduced graph, whereas Constructions (b) and (d) can only be applied to reduced regular graphs of degree $\frac{n}{2}$. On the other hand Constructions (b) and (c) produce reduced regular graphs of degree half the number of vertices, whilst Constructions (a) and (d) produce non-regular graphs. In fact, a stronger property holds for the outcome of Construction (c).

Definition 2 We say that a graph $G$ is 1-closed if it is reduced and for any column of its adjacency matrix there is another column such that the two columns add up to the all-one vector 1 .

The following results follow straightforwardly from the definitions.

Proposition 1 Let $G$ be a graph with $n$ vertices.

(i) If $G$ is reduced, then $G^{c}$ is 1-closed.

(ii) If $G$ is 1 -closed, then $G$ is regular of degree $\frac{n}{2}$.

(iii) If $G$ is 1-closed, then so is $G^{b}$.

(iv) If $G$ is 1-closed, then $G^{b}$ and $G^{c}$ are isomorphic.

(v) If $G$ is 1-closed, then $G^{a}$ and $G^{d}$ are isomorphic.

Although the four constructions are different they often give the same outcome. For example Constructions (b) and (c) produce isomorphic graphs if they are applied to a 1-closed graph, and the same is true for Constructions (a) and (d). Another example is the following lemma. 
Lemma 1 Let $G$ be a reduced graph. Then $\left(G^{a}\right)^{c}$ and $\left(G^{c}\right)^{b}$ are isomorphic.

Proof Let $A$ be the adjacency matrix of $G$ then $\left(G^{a}\right)^{c}$ has adjacency matrix

\begin{tabular}{|c|c|c|c|c|c|c|c|c|c|}
\hline & 1 & 2 & 3 & 1 & & 6 & 7 & 89 & 10 \\
\hline 1 & $A$ & $A$ & $\mathbf{0}$ & $\mathbf{0}$ & $\bar{A}$ & $A$ & 1 & \begin{tabular}{l|l}
1 & 1
\end{tabular} & 0 \\
\hline 2 & $A$ & $A$ & 1 & 0 & $\bar{A}$ & $\bar{A}$ & 0 & \begin{tabular}{l|l}
1 & 1
\end{tabular} & 0 \\
\hline 5 & 0 & 1 & 0 & 1 & 1 & 0 & 1 & \begin{tabular}{l|l}
0 & 1
\end{tabular} & 0 \\
\hline 4 & 0 & 0 & 1 & 0 & 1 & 1 & 0 & \begin{tabular}{l|l}
1 & 1
\end{tabular} & 0 \\
\hline p & $\bar{A}$ & $\bar{A}$ & 1 & 1 & $A$ & $\bar{A}$ & $\mathbf{0}$ & \begin{tabular}{l|l}
$\mathbf{0}$ & 0 \\
\end{tabular} & 1 \\
\hline 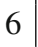 & $\bar{A}$ & $\bar{A}$ & 0 & 1 & $A$ & $A$ & 1 & $\begin{array}{lll}\mathbf{0} & 0\end{array}$ & 1 \\
\hline 7 & 1 & 0 & 1 & 0 & 0 & 1 & 0 & 10 & 1 \\
\hline & 1 & 1 & 0 & 1 & 0 & 0 & 1 & \begin{tabular}{l|l}
0 & 0
\end{tabular} & 1 \\
\hline & 1 & 1 & 1 & 1 & 0 & 0 & 0 & \begin{tabular}{l|l}
0 & 0
\end{tabular} & 1 \\
\hline & & $\mathbf{0}$ & 0 & 0 & & 1 & 1 & & \\
\hline
\end{tabular}

If the rows and columns are re-ordered as indicated by the numbers we get the following matrix:

\begin{tabular}{|c|c|c|c|c|c|c|c|c|c|}
\hline & & 5 & 9 & & & & 8 & & \\
\hline 2 & $A$ & $\bar{A}$ & 1 & \begin{tabular}{|l|}
$\mathbf{0}$ \\
\end{tabular} & $A$ & $\bar{A}$ & 1 & 0 & \\
\hline & $\bar{A}$ & $A$ & 0 & 1 & $\bar{A}$ & $A$ & 0 & 1 & \\
\hline & 1 & 0 & 0 & 1 & 1 & 0 & 0 & 1 & \\
\hline 4 & $\mathbf{0}$ & 1 & 1 & 0 & 0 & 1 & 1 & 0 & 1 \\
\hline 1 & $A$ & $\bar{A}$ & 1 & $\mathbf{0}$ & $A$ & $\bar{A}$ & 1 & $\mathbf{0}$ & $\mathbf{0}$ \\
\hline 6 & $\bar{A}$ & $A$ & 0 & 1 & $\bar{A}$ & $A$ & 0 & 1 & 0 \\
\hline 3 & 1 & 0 & 0 & 1 & 1 & 0 & 0 & 1 & 0 \\
\hline & 0 & 1 & 1 & 0 & 0 & 1 & 1 & 0 & 0 \\
\hline & 1 & 1 & 1 & 1 & 0 & $\mathbf{0}$ & 0 & 0 & 0 \\
\hline & $\mathbf{0}$ & $\mathbf{0}$ & 0 & 0 & & 1 & 1 & 1 & \\
\hline
\end{tabular}

which is the adjacency matrix of $\left(G^{c}\right)^{b}$.

By Proposition 1, $G^{c}$ is $\mathbf{1}$-closed and hence $\left(G^{c}\right)^{c}=\left(G^{c}\right)^{b}$. Therefore:

Corollary 1 If $G$ is a reduced graph, then $\left(G^{a}\right)^{c}$ and $\left(G^{c}\right)^{c}$ are isomorphic.

For the even case, one can start with $G=K_{2}$ of rank 2 which is 1-closed. For each $k \in$ $\left\{0,1,2, \ldots, \frac{r-2}{2}\right\}$, one can first apply $k$ times Construction (c) (or (b), which gives the same outcome), and then apply $\frac{r}{2}-1-k$ times Construction (a) (or (d) once, followed by $\frac{r}{2}-2-k$ times Construction (a)). In this way one finds $\frac{r}{2}$ non-isomorphic reduced graphs of rank $r$ on $n(r)=2^{(r+2) / 2}-2$ vertices if $r$ is even.

For the odd case, we start with $G=K_{3}$ of rank 3. For each $k \in\left\{0,1,2, \ldots, \frac{r-3}{2}\right\}$ one can first apply $k$ times Construction (c), and then apply $\frac{r-3}{2}-k$ times Construction (a). In this way one finds $\frac{r-1}{2}$ non-isomorphic reduced graphs of rank $r$ on $n(r)=5 \cdot 2^{(r-3) / 2}-2$ vertices. Note that if $G$ is $K_{3}$, then $G^{c}$ is the complement of the cube. Therefore these graphs can also be obtained by use of Constructions (a) and (b), if we start with $K_{3}$ or the complement of the cube. So we can conclude that the new constructions give no new reduced graphs of rank $r$ with $n(r)$ vertices. 


\section{Linear extensions}

Let $G$ be a reduced graph with $n$ vertices and adjacency matrix $A$ of $\operatorname{rank} r$. Then $A$ contains a principle $r \times r$ submatrix $B$ of rank $r$ which corresponds to an induced subgraph $H$ of $G$. We call $G$ a linear extension of $H$. We have

$$
A=\left[\begin{array}{cc}
B & Y \\
Y^{\top} & Y^{\top} B^{-1} Y
\end{array}\right]=\left[\begin{array}{c}
B \\
Y^{\top}
\end{array}\right] B^{-1}\left[\begin{array}{ll}
B & Y
\end{array}\right] .
$$

Observe that a zero column or two identical columns in [ $\left.\begin{array}{ll}B & Y\end{array}\right]$ would imply that $G$ is not reduced. Therefore $\left[\begin{array}{ll}B & Y\end{array}\right]$ has at most $2^{n}-1$ columns, so $m(r) \leq 2^{n}-1$.

The above structure of $A$ can be used to design a method to derive all linear extensions of a given graph of full rank $r$. This method is also described in a slightly different form in [1]:

Step 1 Let $H$ be a graph with $r$ vertices and adjacency matrix $B$ of full rank $r$. Check for all $(0,1)$-vectors $\boldsymbol{v} \neq \mathbf{0}$ whether $\boldsymbol{v}^{\top} B^{-1} \boldsymbol{v}=0$ and keep the vector $\boldsymbol{v}$ if so. Let $\left(\boldsymbol{v}_{1}, \boldsymbol{v}_{2}, \ldots, \boldsymbol{v}_{k}\right)$ be the list of such vectors. Clearly this list contains all columns of $B$. Assume without loss of generality that $B=\left[\begin{array}{llll}\boldsymbol{v}_{1} & \boldsymbol{v}_{2} & \cdots & \boldsymbol{v}_{r}\end{array}\right]$.

Step 2 Construct a graph $\mathcal{G}$ on the vertex set $\left\{\boldsymbol{v}_{1}, \boldsymbol{v}_{2}, \ldots, \boldsymbol{v}_{k}\right\}$ as follows. For each two distinct indices $i$ and $j$, put an edge from $\boldsymbol{v}_{i}$ to $\boldsymbol{v}_{j}$ if and only if $\boldsymbol{v}_{i}^{\top} B^{-1} \boldsymbol{v}_{j} \in\{0,1\}$. Notice that any of the vertices $\boldsymbol{v}_{1}, \boldsymbol{v}_{2}, \ldots, \boldsymbol{v}_{r}$ is adjacent to all other vertices, because $B^{-1} \boldsymbol{v}_{i}=\boldsymbol{e}_{i}$ for $i \in\{1, \ldots, r\}$. For a clique $C$ that contains $\boldsymbol{v}_{1}, \boldsymbol{v}_{2}, \ldots, \boldsymbol{v}_{r}$, construct a matrix $X$ that contains the vertices of $C$ as columns. If we suppose that $X=\left[\begin{array}{ll}B & Y\end{array}\right]$, then

$$
A=X^{\top} B^{-1} X=\left[\begin{array}{c}
B \\
Y^{\top}
\end{array}\right] B^{-1}\left[\begin{array}{ll}
B & Y
\end{array}\right]=\left[\begin{array}{cc}
B & Y \\
Y^{\top} & Y^{\top} B^{-1} Y
\end{array}\right]
$$

is the adjacency matrix of a reduced graph of rank $r$ containing $H$ as a subgraph. By determining all cliques of $\mathcal{G}$, we determine all linear extensions of $H$.

We will use this method in the next section for the special cases $H=\frac{r}{2} K_{2}$, and $H=$ $K_{3}+\frac{r-3}{2} K_{2}$.

\section{Main result}

Let $m$ be even. Consider the following $m \times m$ matrix pattern.

$$
M_{m}^{*}=\left[\begin{array}{cccccccccc}
0 & * & * & * & \ldots & \ldots & * & * & * & 1 \\
0 & 0 & * & * & \ldots & \ldots & * & * & 1 & 0 \\
0 & 0 & 0 & \ddots & \ldots & \ldots & . & 1 & 0 & 0 \\
0 & 0 & 0 & \ddots & * & * & . & 0 & 0 & 0 \\
\vdots & \vdots & & & 0 & 1 & . & & \vdots & \vdots \\
\vdots & \vdots & & . & 1 & 0 & & & \vdots & \vdots \\
\vdots & \vdots & . & . & . & & \ddots & & \vdots & \vdots \\
0 & 0 & 1 & 0 & & & & \ddots & \vdots & \vdots \\
0 & 1 & 0 & 0 & \ldots & \ldots & \ldots & \cdots & 0 & 0 \\
1 & 0 & 0 & 0 & \ldots & \ldots & \cdots & \cdots & 0 & 0
\end{array}\right]
$$


Every column of this matrix stands for a type of $(0,1)$-vector where two $(0,1)$-vectors have the same type if they only differ in the $*$-positions. So a column with $k *$-positions defines $2^{k}(0,1)$-vectors. So in total we get $2\left(2^{\frac{m}{2}}-1\right)(0,1)$-vectors. We define $M_{r}$ to be the $r \times\left(2^{(r+2) / 2}-2\right)$ matrix whose columns are the vectors defined by the pattern of $M_{r}^{*}$. Let $R$ be the reverse identity matrix of order $r$, that is the adjacency matrix of $\frac{r}{2} K_{2}$, and define $A_{r}=M_{r}^{\top} R M_{r}$. It is straightforward to check that $A_{r}$ is a symmetric $(0,1)$-matrix with rank $r$ and zeros on the diagonal. So $A_{r}$ defines a reduced graph of rank $r$ on $n(r)=2\left(2^{\frac{r}{2}}-1\right)$ vertices. We call this graph $G_{r}$.

Theorem 1 Let $r$ be even and let $G$ be a linear extension of $\frac{r}{2} K_{2}$. Then $G$ has at most $n(r)$ vertices. In case of equality $G$ is isomorphic to $G_{r}$.

Proof Let $A$ be the adjacency matrix of $G$. Then

$$
A=\left[\begin{array}{cc}
R & Y \\
Y^{\top} & Y^{\top} R^{-1} Y
\end{array}\right]=\left[\begin{array}{c}
R \\
Y^{\top}
\end{array}\right] R^{-1}\left[\begin{array}{ll}
R & Y
\end{array}\right]=Z^{\top} R Z,
$$

where $Z=\left[\begin{array}{ll}R & Y\end{array}\right]$. Now every column $z$ of $Z$ is a $(0,1)$-vector of length $r$ satisfying $z^{\top} R z=0$. Let $V$ be the set of all $(0,1)$-vectors $z \neq \mathbf{0}$ of length $r$ satisfying $z^{\top} R z=0$. Then the columns of $Z$ are elements of $V$. Define a graph $\mathcal{G}$ with vertex set $V$ where two vertices $\boldsymbol{x}$ and $\boldsymbol{y}$ are adjacent if $\boldsymbol{x}^{\top} R \boldsymbol{y} \in\{0,1\}$. Clearly the columns of $Z$ form a clique in $\mathcal{G}$. We now prove that $\mathcal{G}$ can be properly colored with $n(r)$ colors proving that $G$ has at most $n(r)$ vertices.

Let $z=\left[z_{1} \cdots z_{r}\right]^{\top}$ be a column of $Z$. We know that $z^{\top} R z=0$. So if $z_{i}=1$, then $z_{r+1-i}=0$, which means that $z_{i}+z_{r+1-i} \leq 1$. Hence $z$ has at most $\frac{r}{2}$ coefficients that are equal to 1 . We call the set

$$
I_{z}=\left\{i \in\left\{1,2, \ldots, \frac{r}{2}\right\}: z_{i}+z_{r+1-i}=1\right\}
$$

the footprint of $z$. Now if $I \subseteq\left\{1,2, \ldots, \frac{r}{2}\right\}$ and $I$ has cardinality $k>0$, then there are $2^{k}$ different vectors $z \in V$ with footprint $I_{z}=I$. Furthermore, there are $\left(\begin{array}{l}\frac{r}{2} \\ k\end{array}\right)$ different subsets of $\left\{1,2, \ldots, \frac{r}{2}\right\}$ of cardinality $k$. As a consequence,

$$
|V|=\sum_{k=1}^{\frac{r}{2}}\left(\begin{array}{l}
\frac{r}{2} \\
k
\end{array}\right) 2^{k}=3^{\frac{r}{2}}-1 .
$$

Out of the $2^{k}$ different vectors $z \in V$ with footprint $I_{z}=I$, there are $2^{k-1}$ vectors for which $\left|\left\{i \in\left\{1,2, \ldots, \frac{r}{2}\right\}: z_{i}=1\right\}\right|$ is even and $2^{k-1}$ vectors for which $\left|\left\{i \in\left\{1,2, \ldots, \frac{r}{2}\right\}: z_{i}=1\right\}\right|$ is odd. Define

$$
\begin{aligned}
& V(I) \quad=\left\{z \in V: I_{z}=I\right\} \\
& V(I)_{0}=\left\{z \in V(I):\left|\left\{i \in\left\{1,2, \ldots, \frac{r}{2}\right\}: z_{i}=1\right\}\right| \text { is even }\right\} \\
& V(I)_{1}=\left\{z \in V(I):\left|\left\{i \in\left\{1,2, \ldots, \frac{r}{2}\right\}: z_{i}=1\right\}\right| \text { is odd }\right\} .
\end{aligned}
$$

We claim that the sets $V(I)_{0}$ and $V(I)_{1}$ are cocliques in $\mathcal{G}$. For any $\boldsymbol{x}, \boldsymbol{y} \in V(I)$, one can easily check that

$$
\begin{gathered}
\boldsymbol{x}^{\top} R \boldsymbol{y}=\left|\left\{i \in I: \boldsymbol{x}_{i} \neq \boldsymbol{y}_{i}\right\}\right|= \\
\left|\left\{i \in I: \boldsymbol{x}_{i}=1\right\}\right|+\left|\left\{i \in I: \boldsymbol{y}_{i}=1\right\}\right|-2\left|\left\{i \in I: \boldsymbol{x}_{i}=\boldsymbol{y}_{i}=1\right\}\right| .
\end{gathered}
$$


So $\boldsymbol{x}^{\top} R \boldsymbol{y}=0$ if and only if $x=y$. Moreover, if either $\boldsymbol{x}, \boldsymbol{y} \in V(I)_{0}$ or $\boldsymbol{x}, \boldsymbol{y} \in V(I)_{1}$, then $\boldsymbol{x}^{\top} R \boldsymbol{y}$ is even. Hence, if $\boldsymbol{x} \neq \boldsymbol{y}$, then $\boldsymbol{x}^{\top} R \boldsymbol{y} \notin\{0,1\}$. This proves the claim. Now, the vertex set of $\mathcal{G}$ can be partitioned in the right number of cocliques:

$$
\sum_{k=1}^{\frac{r}{2}}\left(\begin{array}{l}
\frac{r}{2} \\
k
\end{array}\right) 2=n(r) .
$$

So the chromatic number of $\mathcal{G}$ is at most $n(r)$, as desired.

Finally we have to prove that all cliques of maximum size in $\mathcal{G}$ correspond with a graph that is isomorphic to $G_{r}$. For this, we make use of the automorphisms of $\frac{r}{2} K_{2}$. Note that one can permute the $\frac{r}{2}$ edges arbitrarily and that every edge can be flipped. These automorphisms imply transformations on elements of $V$ that do not change their mutual inner products: $\phi(\boldsymbol{x})^{\top} R \phi(\boldsymbol{y})=\boldsymbol{x}^{\top} R \boldsymbol{y}$. We show that each maximum clique in $V$ can be transformed to the maximum clique defined by the pattern of $M_{r}^{*}$.

A clique of maximum size should have one vertex in every coclique of the described coloring. $V$ contains $2^{\frac{r}{2}}$ vectors of weight $\frac{r}{2}$, two of which are in the clique. Since all these vectors correspond to all possible ways to assign 0's and 1's to the vertices of $\frac{r}{2} K_{2}$ such that every edge has a 1 and a 0 , these are all isomorphic. So without loss of generality the two vectors of weight $\frac{r}{2}$ in the maximum clique are the characteristic vectors of $\left\{1,2, \ldots, \frac{r}{2}\right\}$ and $\left\{1,2, \ldots, \frac{r}{2}-1, \frac{r}{2}+1\right\}$, since their inner product should be 0 or 1 . Now consider all vectors with footprint $\left\{1,2, \ldots, \frac{r}{2}-1\right\}$. Two of these are in the clique and since the inner product with the previous two vectors should be 0 or 1 , without loss of generality these two vectors are the characteristic vectors of $\left\{1,2, \ldots, \frac{r}{2}-1\right\}$ and $\left\{1,2, \ldots, \frac{r}{2}-2, \frac{r}{2}+2\right\}$. Now consider all vectors in $V$ with footprint $\left\{1,2, \ldots, \frac{r}{2}-2\right\}$, etcetera.

So without loss of generality and by using the automorphisms of $\frac{r}{2} K_{2}$, the columns of $M_{r}^{*}$, where every $*$ is replaced by a 1 are part of the maximum clique. All other vertices of the maximum clique are fixed, since there is only one candidate left in each coclique that can be added to this set. Let $I \subseteq\left\{1,2, \ldots, \frac{r}{2}\right\}$ and let $k$ be the largest element of $I$. Consider the vectors with footprint $I$. Then the only two vectors in $V$ with footprint $I$ that can be in the clique are the characteristic vectors of $I$ and $I \cup\{r-k+1\} \backslash\{k\}$. The resulting maximum clique is the one defined by the pattern of $M_{r}^{*}$.

Theorem 2 Let $r \geq 5$ be odd and let $G$ be a linear extension of $K_{3}+\frac{r-3}{2} K_{2}$. Then $G$ has at most $n(r)$ vertices and there is a unique graph for which equality holds.

Proof Let $A$ be the adjacency matrix of $G$, let $R$ be the reverse identity matrix of order $r-3$, and let $\Delta$ be the adjacency matrix of the triangle $K_{3}$. Then

$$
N=\left[\begin{array}{ll}
R & O \\
O & \Delta
\end{array}\right]
$$

is the adjacency matrix of $K_{3}+\frac{r-3}{2} K_{2}$ and $A=Z^{\top} N^{-1} Z$ for some $r \times n(0,1)$-matrix $Z$. Let $V$ be the set of all $(0,1)$-vectors $\boldsymbol{z} \neq \mathbf{0}$ of length $r$ satisfying $z^{\top} N^{-1} z=0$, and define $\mathcal{G}$ to be the graph with vertex set $V$ where two vertices $\boldsymbol{x}$ and $\boldsymbol{y}$ are adjacent if $\boldsymbol{x}^{\top} N^{-1} \boldsymbol{y} \in\{0,1\}$. Then clearly the columns of $Z$ form a clique in $\mathcal{G}$. We shall prove that $\mathcal{G}$ can be properly colored with $n(r)$ colors proving that $G$ has at most $n(r)$ vertices. Let $z=\left[\begin{array}{l}x \\ y\end{array}\right] \in V$ with $\boldsymbol{y} \in\{0,1\}^{3}$ and $\boldsymbol{x} \in\{0,1\}^{r-3}$, then $\boldsymbol{z}$ is a $(0,1)$-vector satisfying $\boldsymbol{z}^{\top} N^{-1} z=\boldsymbol{x}^{\top} R \boldsymbol{x}+\frac{1}{2} \boldsymbol{y}^{\top}(\Delta-I) \boldsymbol{y}=0$, which can only happen if $\boldsymbol{x}^{\top} R \boldsymbol{x}=0$ and $\boldsymbol{y}^{\top}(\Delta-I) \boldsymbol{y}=0$. So 


$$
\boldsymbol{y} \in\left\{\boldsymbol{y}_{0}=\left[\begin{array}{l}
0 \\
0 \\
0
\end{array}\right], \boldsymbol{y}_{1}=\left[\begin{array}{l}
0 \\
1 \\
1
\end{array}\right], \boldsymbol{y}_{2}=\left[\begin{array}{l}
1 \\
0 \\
1
\end{array}\right], \boldsymbol{y}_{3}=\left[\begin{array}{l}
1 \\
1 \\
0
\end{array}\right]\right\}
$$

and hence $V$ has $4 \cdot 3^{\frac{r-3}{2}}-1$ elements. Let $W$ be the set of all $(0,1)$-vectors $\boldsymbol{x} \neq \mathbf{0}$ of length $r-3$ satisfying $\boldsymbol{x}^{\top} R \boldsymbol{x}=0$. For a vector $\boldsymbol{x}=\left[x_{1} \cdots x_{r-3}\right]^{\top} \in W$, we define its footprint $I x$ as

$$
I_{\boldsymbol{x}}=\left\{i \in\left\{1,2, \ldots, \frac{r-3}{2}\right\}: x_{i}+x_{r-2-i}=1\right\} .
$$

For $I \subseteq\left\{1,2, \ldots, \frac{r-3}{2}\right\}, I \neq \emptyset$ we define the following sets:

$$
\begin{aligned}
& W(I)=\{\boldsymbol{x} \in W: I x=I\} ; \\
& W(I)_{0}=\left\{\boldsymbol{x} \in W(I):\left|\left\{i \in\left\{1,2, \ldots, \frac{r-3}{2}\right\}: x_{i}=1\right\}\right| \text { is even }\right\} ; \\
& W(I)_{1}=\left\{\boldsymbol{x} \in W(I):\left|\left\{i \in\left\{1,2, \ldots, \frac{r-3}{2}\right\}: x_{i}=1\right\}\right| \text { is odd }\right\} .
\end{aligned}
$$

Now the following partition of $V$ defines a proper coloring of $\mathcal{G}$.

First the three sets

$$
\left\{\left[\begin{array}{c}
0 \\
y_{1}
\end{array}\right]\right\},\left\{\left[\begin{array}{c}
0 \\
y_{2}
\end{array}\right]\right\},\left\{\left[\begin{array}{c}
0 \\
y_{3}
\end{array}\right]\right\} \text {. }
$$

Next, for every $I \subseteq\left\{1,2, \ldots, \frac{r-3}{2}\right\}, I \neq \emptyset$ we take the following five sets:

$$
\begin{aligned}
& \left\{\left[\begin{array}{c}
\boldsymbol{x} \\
\boldsymbol{y}_{0}
\end{array}\right]: x \in W(I)_{0}\right\},\left\{\left[\begin{array}{l}
\boldsymbol{x} \\
\boldsymbol{y}_{0}
\end{array}\right]: x \in W(I)_{1}\right\}, \\
& \left\{\left[\begin{array}{c}
\boldsymbol{x} \\
\boldsymbol{y}_{1}
\end{array}\right]: \boldsymbol{x} \in W(I)_{0}\right\} \cup\left\{\left[\begin{array}{c}
\boldsymbol{x} \\
\boldsymbol{y}_{2}
\end{array}\right]: \boldsymbol{x} \in W(I)_{1}\right\}, \\
& \left\{\left[\begin{array}{c}
\boldsymbol{x} \\
\boldsymbol{y}_{2}
\end{array}\right]: \boldsymbol{x} \in W(I)_{0}\right\} \cup\left\{\left[\begin{array}{c}
\boldsymbol{x} \\
\boldsymbol{y}_{3}
\end{array}\right]: \boldsymbol{x} \in W(I)_{1}\right\}, \\
& \left\{\left[\begin{array}{c}
\boldsymbol{x} \\
\boldsymbol{y}_{3}
\end{array}\right]: \boldsymbol{x} \in W(I)_{0}\right\} \cup\left\{\left[\begin{array}{c}
\boldsymbol{x} \\
\boldsymbol{y}_{1}
\end{array}\right]: \boldsymbol{x} \in W(I)_{1}\right\},
\end{aligned}
$$

Similarly as in the previous proof it can be checked that the $5 \cdot\left(2^{\frac{r-3}{2}}-1\right)+3=n(r)$ sets are cocliques in $\mathcal{G}$. Thus we found a proper coloring with $n(r)$ colors, as promised.

To show that all cocliques in $\mathcal{G}$ of size $n(r)$ lead to isomorphic graphs, we will also use the following proper coloring of $\mathcal{G}$.

First the three sets

$$
\left\{\left[\begin{array}{c}
0 \\
y_{1}
\end{array}\right]\right\},\left\{\left[\begin{array}{c}
0 \\
y_{2}
\end{array}\right]\right\},\left\{\left[\begin{array}{c}
0 \\
y_{3}
\end{array}\right]\right\} \text {. }
$$

Next, for every $I \subseteq\left\{1,2, \ldots, \frac{r-3}{2}\right\}, I \neq \emptyset$ we take the following five sets:

$$
\begin{aligned}
& \left\{\left[\begin{array}{c}
\boldsymbol{x} \\
\boldsymbol{y}_{0}
\end{array}\right]: x \in W(I)_{0}\right\},\left\{\left[\begin{array}{l}
\boldsymbol{x} \\
\boldsymbol{y}_{0}
\end{array}\right]: x \in W(I)_{1}\right\}, \\
& \left\{\left[\begin{array}{c}
\boldsymbol{x} \\
\boldsymbol{y}_{1}
\end{array}\right]: \boldsymbol{x} \in W(I)_{0}\right\} \cup\left\{\left[\begin{array}{c}
\boldsymbol{x} \\
\boldsymbol{y}_{3}
\end{array}\right]: x \in W(I)_{1}\right\}, \\
& \left\{\left[\begin{array}{c}
\boldsymbol{x} \\
\boldsymbol{y}_{3}
\end{array}\right]: \boldsymbol{x} \in W(I)_{0}\right\} \cup\left\{\left[\begin{array}{c}
\boldsymbol{x} \\
\boldsymbol{y}_{2}
\end{array}\right]: \boldsymbol{x} \in W(I)_{1}\right\}, \\
& \left\{\left[\begin{array}{c}
\boldsymbol{x} \\
\boldsymbol{y}_{2}
\end{array}\right]: \boldsymbol{x} \in W(I)_{0}\right\} \cup\left\{\left[\begin{array}{c}
\boldsymbol{x} \\
\boldsymbol{y}_{1}
\end{array}\right]: \boldsymbol{x} \in W(I)_{1}\right\} .
\end{aligned}
$$


Now suppose we have a clique in $\mathcal{G}$ of size $n(r)$. Then this clique contains one vertex of each of the cocliques of the two proper colorings. So it contains the three vectors

$$
\left[\begin{array}{c}
\mathbf{0} \\
y_{1}
\end{array}\right],\left[\begin{array}{c}
\mathbf{0} \\
y_{2}
\end{array}\right] \text { and }\left[\begin{array}{c}
\mathbf{0} \\
y_{3}
\end{array}\right] \text {. }
$$

The clique also contains $2 \cdot\left(2^{\frac{r-3}{2}}-1\right)$ vertices of the form

$$
\left\{\left[\begin{array}{c}
x \\
y_{0}
\end{array}\right]: x \in W(I)\right\} \text {. }
$$

Like in the proof of Theorem 1, it follows that without loss of generality we can take the vertices for which $\boldsymbol{x}$ is a vector that fits the pattern of $M_{r-3}^{*}$. Next for every $I \subseteq$ $\left\{1,2, \ldots, \frac{r-3}{2}\right\}, I \neq \emptyset$ we have three vectors

$$
\left[\begin{array}{l}
x_{1} \\
y_{1}
\end{array}\right],\left[\begin{array}{l}
x_{2} \\
y_{2}
\end{array}\right] \text { and }\left[\begin{array}{l}
x_{3} \\
y_{3}
\end{array}\right]
$$

in the clique. Because the clique contains a vertex in each color class of both given colorings, it follows that either $\boldsymbol{x}_{1}, \boldsymbol{x}_{2}, \boldsymbol{x}_{3} \in W(I)_{0}$ or $\boldsymbol{x}_{1}, \boldsymbol{x}_{2}, \boldsymbol{x}_{3} \in W(I)_{1}$. It can be verified that without loss of generality $\boldsymbol{x}_{1}, \boldsymbol{x}_{2}$ and $\boldsymbol{x}_{3}$ are all three equal to the characteristic vector of $I$.

\section{Concluding remarks}

In the previous section we considered maximum linear extensions of $\frac{r}{2} K_{2}$ and $\frac{r-3}{2} K_{2}+K_{3}$. If the conjecture of Akbari, Cameron and Khosrovshahi is correct, then a linear extension of any other graph of full rank $r$ has at most $n(r)$ vertices. For a linear extension of a graph $H$ with adjacency matrix $B$ to be large, we need many vectors $\boldsymbol{v}$ for which $\boldsymbol{v}^{\top} B^{-1} \boldsymbol{v}=0$. For most choices of $H$ the number of such vectors is rather small. Especially if $B^{-1}$ contains few zeros, one doesn't expect many such vectors. If $H$ is the complete graph $K_{r}$, for example, the only such vectors are the columns of $B$. Intuitively one expects that the two choices of $H$ considered in this paper have the maximum number of vertices in their maximum linear extensions. Proving this intuitive result, would prove the conjecture. Therefore we strongly believe that the conjecture is true.

The following results are straightforward consequences of the constructions of Sect. 2.

Proposition 2 If $G$ is a linear extension of $H$, then $G^{a}$ is a linear extension of $H+K_{2}$.

As a consequence, it follows that the graphs $G_{r}$ with $n(r)$ vertices, mentioned in Theorem 1 and 2, are isomorphic to the ones obtained by repeatedly applying Construction (a) starting from $K_{2}$ if $r$ is even, or $K_{3}$ if $r$ is odd. The other constructions are linear extensions of graphs $H$ different from $\frac{r}{2} K_{2}$ and $\frac{r-3}{2} K_{2}+K_{3}$.

Proposition 3 Let $G$ be a linear extension of $H=(V, E)$, and let $H^{\prime}=\left(V, E^{\prime}\right)$ with $V^{\prime}=V \cup\left\{v_{1}, v_{2}\right\}$ and $E^{\prime}=E \cup\left\{\left\{v, v_{1}\right\}: v \in V\right\} \cup\left\{v_{1}, v_{2}\right\}$. Then $G^{a}, G^{b}, G^{c}$ and $G^{d}$ (if defined) are linear extensions of $H^{\prime}$.

If $G$ is a reduced graph of rank $r$, then any induced subgraph of full rank $r$ can be linearly extended to $G$. In particular, for the unique graph $G_{r}$ of Theorem 1, every induced subgraph of full rank $r$ has a linear extension with at least $n(r)$ vertices. This leads to the following result. 
Theorem 3 Let $r$ be even and let $H$ be a graph on $r$ vertices having an adjacency matrix $B$ of the following form.

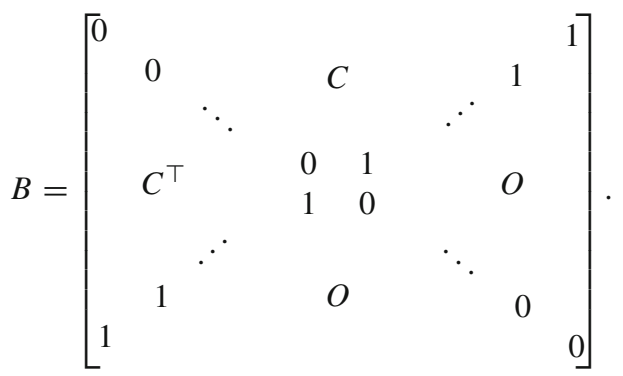

Then $H$ has rank $r$ and $G_{r}$ is a linear extension of $H$.

Proof Clearly rank $B=r$. Let $A_{r}$ be the adjacency matrix of $G_{r}$, and recall that $R$ is the adjacency matrix of $\frac{r}{2} K_{2}$. Define

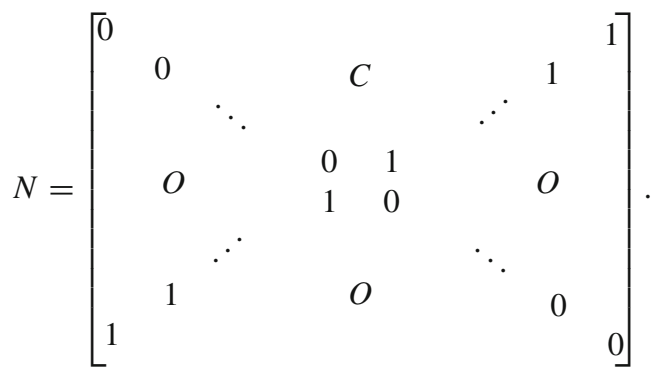

Then $B=N^{\top} R N$. Since each column of $N$ is also a column of $M_{r}$, we get that $H$ is a subgraph of $G_{r}$.

Thus, because of Proposition 3 and Theorem 3, we have many graphs of full rank $r$ whose maximum linear extension has at least $n(r)$ vertices. To show that these linear extensions have at most $n(r)$ vertices could be an important step towards a proof of the conjecture.

Acknowledgment We thank the referees for many important remarks, which lead to a considerable improvement of the manuscript.

Open Access This article is distributed under the terms of the Creative Commons Attribution Noncommercial License which permits any noncommercial use, distribution, and reproduction in any medium, provided the original author(s) and source are credited.

\section{References}

1. Akbari S., Cameron P.J., Khosrovshahi G.B.: Ranks and signatures of adjacency matrices (preprint 2004). http://www.maths.qmw.ac.uk/ pjc/preprints/ranksign.pdf.

2. Godsil C.D., Royle G.F.: Chromatic number and the 2-rank of a graph. J. Combin. Theory Ser. B 81, 142-149 (2001).

3. Kotlov A., Lovász L.: The rank and size of graphs. J. Graph Theory 23, 185-189 (1996). 